\title{
ON FUNDAMENTAL SETS OVER A FINITE FIELD
}

\author{
YOUSEF ABBAS and JOSEPH J. LIANG \\ Department of Mathematics \\ University of South Florida \\ Tampa, Florida 33620 U.S.A.
}

(Received March 3, 1983)

ABSTRACT. A partition over finite field is defined and each equivalence class is constructed and represented by a set called the fundamental set. If a primitive element is used to construct the addition table over these fundamental sets then all additions over the field can be computed. The number of partitions is given for some finite fields.

KEY WORDS AND PHRASES. Finite fiela, primitive polynomial. 1980 MATHEMATICS SUBJECT CLASSIFICATION CODE. 12 C99.

\section{INTRODUCTION}

Throughout, $p, q$ will be fixed but arbitrary primes.

Let $\alpha \in G F\left(p^{n}\right) ; n>1$ and define $A=A_{\alpha}=\{\alpha, \alpha+1, \alpha+2, \ldots, \alpha+(p-1)\}$. If $B \in A_{\alpha}$, then $A_{B}=A_{\alpha}$. Define $2 A_{\alpha}, 3 A_{\alpha}, \ldots,(p-1) A_{\alpha}$ such that $\ell A_{\alpha}=\left\{\ell x / x \in A_{\alpha}\right\}$ $\ell=1,2, \ldots, p-1$, thus $\ell A_{\alpha}=A_{\ell \alpha}$.

LEFINITION.

$$
{ }^{\star} A_{\alpha} \equiv \bigcup_{\ell=1}^{p-1} \ell A_{\alpha}=\bigcup_{l=1}^{p-1} A_{\ell \alpha} .
$$

Note that if $B \in{ }^{*} A_{\alpha}$, then ${ }^{\star}{ }_{A}={ }^{\star}{ }_{A_{B}}$.

DEFInITION. $A_{\alpha}^{\mathrm{p}^{\ell}}=\left\{\mathrm{x}^{\mathrm{p}^{\ell}} / \mathrm{x} \in \mathrm{A}_{\alpha}\right\}$ for $\ell=0,1,2, \ldots$, so, $\mathrm{A}_{\alpha}^{\mathrm{p}^{\ell}}=\alpha^{\mathrm{p}^{\ell}}, \alpha^{\mathrm{p}^{\ell}}+1, \ldots$, $\left.\alpha^{p^{l}}+(p-1)\right\}=A_{\alpha} p^{\ell}$. Since $\alpha^{p^{n}}=\alpha$ for every $\alpha \in G F\left(p^{n}\right)$, we have $A_{\alpha}^{p^{n}}=A_{\alpha}$. Therefore, the values of $\ell$ in the definition can be limited to $0,1,2, \ldots, n-1$.

DEFINITION.

$$
\begin{aligned}
& { }^{\star}{ }_{\mathrm{A}_{\alpha}} \mathrm{p}^{\ell} \equiv{ }^{\star}{ }_{\mathrm{A}_{\alpha}} \mathrm{P}^{\ell}={ }^{\star}\left(\mathrm{A}_{\alpha}^{\mathrm{p}^{l}}\right) \text {. } \\
& \bar{A}_{\alpha}=\bigcup_{\ell=0}^{n-1}{ }^{*}{ }_{\alpha} p^{\ell}
\end{aligned}
$$

DEFINITION.

thus, $\bar{A}_{\alpha}=\left\{a \alpha^{p^{j}}+b \mid a \in z_{p}^{*}, b \in z_{p}\right.$ and $\left.j \in z_{n}\right\}$.

LEMMA 1.1. If $B \in \bar{A}_{\alpha}$, then $\bar{A}_{\alpha}=\bar{A}_{B}$.

PROOF. If $B \in \bar{A}_{\alpha}$, then there exist $a \in z_{p}^{*}, b \in z_{p}$ and $j \in z_{n}$ such that $B=a \alpha p^{j}+b$. This implies $\bar{A}_{B} \subset \bar{A}_{\alpha}$. Since $\left(z_{p},+,.\right)$ is a field and a $\neq 0$ both $a^{-1},-b$ exist. Therefore, $\alpha=a^{-1} p^{p^{n-j}}+a^{-1}(-b)$ which says $\alpha \in \bar{A}_{B}$. Hence $\bar{A}_{\alpha} \subset \bar{A}_{B}$
or $\bar{A}_{\alpha}=\bar{A}_{B}$. 
DEFINITION. A will be called a Fundamental set.

Since $A_{\alpha}^{p^{n}}=A_{\alpha}$ for every $\alpha \in G F\left(p^{n}\right)$, there exists a least positive integer $m \leq n$ such that $\mathrm{A}_{\alpha}^{\mathrm{P}^{\mathrm{m}}}=\mathrm{aA} \mathrm{A}_{\alpha}$ for some $\mathrm{a} \in \mathrm{Z}_{\mathrm{p}}^{*}$. It follows that $\overline{\mathrm{A}}_{\alpha}=\bigcup_{l=0}^{\mathrm{m}-1}{ }^{\star} \mathrm{A}_{\alpha}^{\mathrm{P}^{\ell}}$.

DEFINITION. Let $\alpha, \beta \in G F\left(p^{n}\right)$. We define the relation $\sim$ in $G F\left(p^{n}\right)$ as

$$
\alpha \sim \beta \text { iff } \bar{A}_{\alpha}=\bar{A}_{B}
$$

THEOREM 1.1. From Lemma 1.1. the relation $\sim$ is an equivalence relation. This equivalence relation $\sim$ will partition the field $G F\left(p^{n}\right)$ into equivalence classes $\bar{A}_{\alpha}$ and each class is represented by a fundamental set.

DEFINITION. $\bar{A}_{\alpha}$ will be called a Fundamental class.

Let $\theta$ be a primitive element in $G F\left(p^{n}\right)$. Since, $\theta^{\frac{p^{n}-1}{p-1}}$ is primitive in $Z_{p}$, then for every $a \in z_{p}^{*}$ there exists $k_{a}$, where $1 \leq k_{a} \leq p-1$. So, $k_{a}$ can be determined easily. If the elements of the fundamental set $A_{\alpha}$ are expressed as powers of $\theta$, then $\bar{A}_{\alpha}$ can be expressed from $A_{\alpha}$ as powers of $\theta$. So to calculate the addition table of $\overline{\mathrm{A}}_{\alpha}^{\alpha}$, it is sufficient to have the addition table of $\mathrm{A}_{\alpha}$. Therefore, if $\overline{\mathrm{A}}_{\alpha_{1}}, \overline{\mathrm{A}}_{\alpha_{2}}, \ldots, \overline{\mathrm{A}}_{\alpha_{s}}$, are all the fundamental classes in $G F\left(p^{n}\right)$, it will be enough to tabulate only the addition tables over $A_{\alpha_{1}}, \ldots, A_{\alpha_{s}}$ with respect to $\theta$ to do all the calculations over $G F\left(p^{n}\right)$.

If for some $\alpha \in G F\left(p^{n}\right)$, $m$ is the least positive integer such that $\alpha^{p^{m}}=a \alpha+b$ for some $a \in z_{p}^{*}$ and $b \in z_{p}$, then it is true that $m$ will be the smallest positive integer for every $B \in \bar{A}_{\alpha}$ such that $\beta^{p^{m}}=a B+b^{\prime}$ where $b^{\prime} \in z_{p}$. This will be shown below.

DEFINITION. Let $\alpha \in G F\left(\mathrm{p}^{\mathrm{n}}\right)$, if $\mathrm{m}$ is the least positive integer such that $\alpha^{p^{m}}=a \alpha+b$ for some $a \in z_{p}^{*}, b \in Z_{p}$ then $m$ is called the index of $\alpha$ and $a$ is the coefficient of $\alpha$ and we say $\alpha$ has an index m with coefficient $a$. If $\alpha \in Z_{p}$ we say $\alpha$ has index 0 with coefficient $a=1$.

LEMMA 1.2. If $\alpha$ has index m with coefficient $a$, then every $B \in \bar{A}$ has the same index $m$ and the same coefficient $a$.

PROOF. Let $B \in \bar{A}$ with index $s$ and $B \notin z_{p}$. There exist $\ell \in z_{p}^{*}, \delta \in z_{p}$ and $j \in Z_{n}$ such that $\beta=\ell \alpha^{p^{j}}+\delta$. This implies $\beta^{p^{m}}=\left(\ell \alpha^{p^{j}}+\delta\right)^{p^{m}}=\ell(a \alpha+b)^{p^{j}}+\delta=$ $a B+c$ where $c \in Z_{p}$. Therefore $s \leq m$. But from Lemma 1.1 . we have $\bar{A}_{\alpha}=\bar{A}_{B}$. Hence $\alpha \in \bar{A}$. Therefore $\mathrm{m} \leq \mathrm{s}$, which implies $B$ has index $\mathrm{m}$ with coefficient $a$.

DEFINITION. The fundamental set $A_{\alpha}$ has index $m$ with coefficient $a$ if and only if $m$ is the least positive integer such that $A^{p^{m}}=a A$.

THEOREM 1.2. If $\alpha$ has index $m$ with coefficient $a$, then $A_{\alpha}$ has index $m$ with coefficient a and each fundamental set $A_{\beta} \subset \bar{A}_{\alpha}$ has the same index $m$ with the same coefficient a.

PROOF. Follows from Lemma 1.2 .

From the above theorem we can define the index $\bar{A}_{\alpha}$ to be the index of any element or any fundamental set included in $\overline{\mathbf{A}}_{\alpha}$.

Now, we want to discuss some properties of the index and the coefficient of the fundamental set. 
THEOREM 1.3. In $G F\left(p^{n}\right)$, if $A$ has index $m$ and coefficient a then $m$ divides $\mathrm{n}$ and $\mathrm{a}^{\mathrm{n} / \mathrm{m}}=1$.

PROOF. Let $n=l m+r, 0 \leq r<m$. Since $\alpha^{p^{m}}=a \alpha+b$, for some $b \in z_{p}$, we have $\alpha^{p^{\ell m}}=a^{\ell}(\alpha)+\left(a^{\ell-1}+a^{\ell-2}+\ldots+1\right) b$ and $\alpha=\alpha^{p^{n}}=\left(\alpha^{p^{\ell m}}\right) p^{r}=a^{\ell} \cdot \alpha^{r}+\left(a^{\ell-1}+a^{\ell-2}+\right.$ $\ldots+1) b$. So, $A^{p^{r}}=a^{l} A$ which implies $r=0$ and $a=a^{n / m}=1$.

THEOREM 1.4. Let $A$ be a fundamental set with index $m$ and coefficient a. If $\mathrm{A}^{\mathrm{P}^{\ell}}=\mathrm{bA}, \mathrm{b} \in \mathrm{z}_{\mathrm{p}}^{*}$, then $\mathrm{m}$ divides $\ell$ and $\mathrm{a}^{\ell / \mathrm{m}}=\mathrm{b}$.

PROOF. Since $m$ is the index of $A$, we have $m \leq \ell$. Let $\ell=k m+r, 0 \leq r<m$. If $\alpha \in A$, then there exist $\delta, \delta^{\prime} \in z_{p}$ such that $\alpha^{\mathrm{p}^{\mathrm{m}}}=a \alpha+\delta$ and $\alpha^{\mathrm{p}^{\ell}}=\mathrm{b} \alpha+\delta^{\prime}$. But $\mathrm{b} \alpha+\delta^{\prime}=\left(\alpha^{\mathrm{p}^{\mathrm{km}}}\right)^{\mathrm{p}^{\mathrm{r}}}=\left(\mathrm{a}^{\mathrm{k}} \alpha+\delta^{*}\right)^{\mathrm{p}^{\mathrm{r}}}+\delta^{\prime}=\mathrm{a}^{\mathrm{k}} \alpha^{\mathrm{p}^{\mathrm{r}}}+\delta^{\prime \prime}$. This implies $\mathrm{r} \geq \mathrm{m}$. Therefore $\mathrm{r}=0$. Hence $\mathrm{m} / \ell$ and $\mathrm{a}^{\ell / \mathrm{m}}=\mathrm{b}$. Note: Since all finite fields of the same order are isomorphic, if $\alpha_{1} \in \mathrm{GF}_{1}\left(\mathrm{p}^{\mathrm{n}}\right)$ having index $\mathrm{m}$ with coefficient $a$, is the image of $\alpha_{2} \in \mathrm{GF}_{2}\left(\mathrm{p}^{\mathrm{n}}\right)$ under an isomorphism $\sigma$, then $\alpha_{2}$ has index $m$ with coefficient a. Therefore; $G F_{1}\left(p^{n}\right)$ and $G F_{2}\left(p^{n}\right)$ have the same partitions with respect to the equivalence relation $\sim$.

EXAMPLE: Let $F=G F\left(5^{2}\right)$ and $\theta$ be a primitive element in $F$ such that $\theta$ satisfies the primitive polynomial [ indexing polynomial], $P(x)=x^{2}+4 x+2$. The field $F$ has two equivalence classes, $Z_{5}$ and $\bar{A}_{\theta} . A_{\theta}=\left\{\theta, \theta+1=\theta^{22}, \theta+2=\theta^{15}, \theta+3=\theta^{2}\right.$, $\theta+4=\theta^{17}$. So, we have $\theta \rightarrow \theta^{22^{\theta}}+\theta^{15} \rightarrow \theta^{2} \rightarrow \theta^{17}$. Since $\theta^{6}=2$, then $4=\theta^{12}$ and $3=\theta^{18}$. Therefore,

$$
\begin{aligned}
& 2 A_{\theta}=\left\{\theta^{7}, \theta^{7}+2=\theta^{4}, \theta^{7}+4=\theta^{21}, \theta^{7}+1=\theta^{8}\right\}, \\
& 3 A_{\theta}=\left\{\theta^{19}, \theta^{19}+3=\theta^{16}, \theta^{19}+1=\theta^{10}, \theta^{19}+4=\theta^{21}, \theta^{19}+2=\theta^{6}\right\}, \\
& 4 A_{\theta}=\left\{\theta^{13}, \theta^{13}+4=\theta^{10}, \theta^{13}+3=\theta^{3}, \theta^{13}+2=\theta^{14}, \theta^{13}+1=\theta^{5}\right\}
\end{aligned}
$$

and $A_{\theta}^{5}=4 A_{\theta}$.

2. SOLUTIONS OF EQUATIONS OF THE FORM $\mathrm{A}^{\mathrm{P}^{\mathrm{m}}}=\mathbf{a A}$.

To study the fundamental sets in $G F\left(p^{n}\right)$ with index $m<n$, we have to study the solutions of: (a) $x^{p^{m}}=a x+\delta ; a \in z_{p}^{*}, a \neq 1, \delta \in z_{p}$ and (b) $x^{p^{m}}=x+\delta$; $\delta \in z_{p}^{*}$ in $G F\left(p^{n}\right)$. Note: If $\delta=0$ in (b), then all the elements of the subfield $G F\left(p^{m}\right)$ will satisfy (b).

We will now consider the solutions of

$$
x^{p^{m}}=a x+\delta
$$

where $a \in z_{p}^{*}, a \neq 1$ and $\delta \in z_{p}$.

LEMMA 2.1. Equation (2.1) has a solution $\alpha \notin z_{p}$ with index $m$ only if $m$ divides $\mathrm{n}$ and $\mathrm{a}^{\mathrm{n} / \mathrm{m}}=1$.

The proof is a direct application of Theorem 1.3 and Theorem $\mathrm{m}^{1.4}$.

LEMMA 2.2. In equation (2.1), for $y=x+\frac{\delta}{a-1}$, we have $y^{p^{m}}=a y$.

PROOF.

$$
\begin{aligned}
y^{p^{m}} & =\left(x+\frac{\delta}{a-1}\right)^{p^{m}}=x^{p^{m}}+\frac{\delta}{a-1} \\
& =a x+\delta+\frac{\delta}{a-1}=a\left(y-\frac{\delta}{a-1}\right)+\frac{a \delta}{a-1} \\
& =a y .
\end{aligned}
$$


Therefore, to study the solutions of (2.1), it is sufficient to study the solutions of $\mathrm{y}^{\mathrm{P}^{\mathrm{m}}}=$ ay.

LEMMA 2.3. $\quad x=\frac{\delta}{1-a}$ is a solution of equation (2.1).

If $\theta$ is a primitive element in $G F\left(p^{n}\right)$, then the following statements are true:

(a) $\theta^{\frac{p^{n}-1}{p-1}} \in z_{p}^{*}$

(b) For every $a \in Z_{p}^{*}$ there exists an integer $k_{a}$ such that $a=\theta^{\frac{p^{n}-1}{p-1} k_{a}}$, where $0 \leq \mathrm{k}_{\mathrm{a}}<\mathrm{p}-1$. If $\mathrm{a} \neq 1, \mathrm{k}_{\mathrm{a}} \neq 0$.

LEMMA 2.4. For every $m$ such that $m$ divides $n$ and $\frac{n}{m} \mid p-1$, then $\frac{p^{n}-1}{p^{m}-1} \cdot \frac{1}{n / m}$ is an integer.

PROOF. Let $\frac{n}{m}=k$. So, $p \equiv 1(\bmod k)$ and $\frac{p^{n}-1}{p^{m}-1}=\frac{\left(p^{m}\right)^{k}-1}{p^{m}-1}=\left(p^{m}\right)^{k-1}+\left(p^{m}\right)^{k-2}+$ $\ldots+1$. Since $p \equiv 1(\bmod k)$, we have $p^{j} \equiv 1(\bmod k)$ for $j=1,2, \ldots$. Therefore, $\sum_{j=0}^{k-1}\left(p^{m}\right)^{j} \equiv 0(\bmod k)$. Hence $\frac{n}{m} \mid \frac{p^{n}-1}{p^{m}-1}$.

LEMMA 2.5. If $a^{n / m}=1, a \neq 1$ and $\frac{n}{m} \mid p-1$, then $\frac{p^{n}-1}{p^{m}-1} \cdot \frac{k_{a}}{p-1}$ is an integer.

PROOF. $\frac{p^{n}-1}{p^{m}-1} \cdot \frac{k}{p-1}=\frac{p^{n}-1}{p^{m}-1} \cdot \frac{1}{n / m} \cdot \frac{(n / m)^{k} a}{p-1}$.

But, from Lemma $2.4 \frac{p^{n}-1}{p^{n}-1} \cdot \frac{1}{p^{m}-1}$ is an integer. Also, $a=\theta \frac{p^{n}-1}{p-1} \cdot k_{a}$ implies $a^{n / m}=\theta^{\frac{p^{n}-1}{p-1} \cdot k_{a} \cdot \frac{n}{m}}=1$. Therefore, $\frac{p^{n}-1}{p-1} k_{a} \cdot \frac{n}{m} \equiv 0 \bmod \left(p^{n}-1\right)$. So $p-1 \mid k_{a} \cdot \frac{n}{m}$.

THEOREM 2.1. Given $\mathrm{x}^{\mathrm{p}^{\mathrm{m}}}=\mathrm{ax}$

where $m \mid n, a \neq 1$ and $O(a)=\frac{n}{m}$, then

(a) Equation (2.2) has solution $\alpha$ in $G F\left(p^{n}\right)$ and $\alpha \in z_{p}$.

(b) If $\alpha$ is a solution of (2.2) where $\alpha=\theta^{r}, \theta$ is primitive in $G F\left(p^{n}\right)$, then

$$
r \equiv \frac{p^{n}-1}{p^{m}-1} \cdot \frac{k_{a}}{p-1} \quad\left(\bmod \frac{p^{n}-1}{p^{m}-1}\right) .
$$

(c) $x=0$ is the only solution of (2.2) in $z_{p}$.

PROOF.

(a) If $x \in z_{p}$, then $x^{p^{m}}=x=a x$. Therefore $x=0$.

(b) Since $x^{p^{m}}=$ ax and $a \neq 1$, then $x \notin G F\left(p^{m}\right)$.

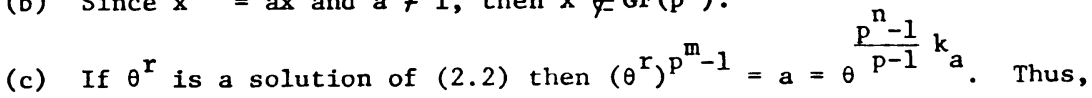

$$
r\left(p^{m}-1\right) \equiv \frac{p^{n}-1}{p-1} k_{a}\left(\bmod p^{n}-1\right)
$$

which implies $r \equiv \frac{p^{n}-1}{p^{m}-1} \cdot \frac{k_{a}}{p-1}\left(\bmod \frac{p^{n}-1}{p^{m}-1}\right)$.

It follows from Lemma 2.5 that the above congruence is meaningful. 
THEOREM 2.2. For every $r \equiv \frac{p^{n}-1}{p^{m}-1} \cdot \frac{k_{a}}{p-1}\left(\bmod \frac{p^{n}-1}{p^{m}-1}\right), \theta^{r}$ is a solution of

PROOF. Let $r=\frac{p^{n}-1}{p^{m}-1} \cdot \frac{k a}{p-1}+j \frac{p^{n}-1}{p^{m}-1} ; j=0,1,2, \ldots$ then

$\left(\theta^{r}\right)^{p^{m}-1}=\theta^{\frac{p^{n}-1}{p-1} \cdot k} a \cdot\left(\theta^{p^{n}-1}\right)^{j}=a \cdot 1=a$. So, $\left(\theta^{r}\right)^{p^{m}}=a \theta$.

Now consider the solutions of

$$
x^{p^{m}}=a x+\delta
$$

where $a \neq 1, a \in z_{p}^{*}, \delta \in z_{p}, m \mid n$ and $0(a)=\frac{n}{m}$. From Lemma 2.2, Theorem 2.1 and Theorem 2.2, we have $\theta^{r}+\frac{\delta}{1-a}$ is a solution of $(2.3)$, where $r \equiv \frac{p^{n}-1}{p^{m}-1} \cdot \frac{k_{a}}{p-1} \quad\left(\bmod \frac{p^{n}-1}{p^{m}-1}\right)$ and $\frac{\delta}{1-a}$ is the only solution in $z_{p}$.

THEOREM 2.3. Let $A$ be a fundamental set in $G F\left(p^{n}\right)$ with index $m$ and coefficient $a \neq 1$. If $A$ is not included in any proper subfield of $G F\left(p^{n}\right)$, then $O(a)=\frac{n}{m}$ and $\frac{\mathrm{n}}{\mathrm{m}} \mid(\mathrm{p}-1)$.

PROOF. Let $O(a)=d$ and $\alpha \in A$. It follows $d \mid \frac{n}{m}$ and $\alpha$ has an index m with coefficient a. Thus, $\alpha^{p^{m}}=a \alpha+\delta$ for some $\delta \in Z_{p}$. Hence $\alpha^{p^{d m}}=a^{d} \alpha+\left(\alpha^{d-1}+\alpha^{d-2}+\ldots+1\right) \delta=\alpha$. Therefore $\alpha \in G F\left(p^{d m}\right)$, which Implies $G F\left(p^{d m}\right)=G F\left(p^{n}\right)$ and $d=\frac{n}{m}$.

COROLLARY 2.1. Let $\mathrm{x}^{\mathrm{p}^{\mathrm{m}}}=\mathrm{ax}+\delta$

such that $a \neq 1, \delta \in z_{p}, m \mid n, a^{\frac{n}{m}}=1$ and $O(a)=l$, then equation (2.4) has solutions in $G F\left(p^{n}\right)$ and all the solutions are included in $G F\left(p^{l m}\right)$.

PROOF. $G F\left(p^{\ell m}\right)$ is a subfield of $G F\left(p^{n}\right)$. Equation (2.4) satisfies the conditions of Theorem 2.1 over $G F\left(p^{\ell m}\right)$ and if $\alpha$ is a solution, then $\alpha^{\mathrm{p}^{\ell m}}=\mathrm{a}^{\ell} \alpha+\left(\mathrm{a}^{\ell-1}+\mathrm{a}^{\ell-2}+\ldots+1\right) \delta=\alpha+0=\alpha$. Therefore $\alpha \in \mathrm{GF}\left(\mathrm{p}^{\ell \mathrm{m}}\right)$.

Note: If $\theta$ is a primitive element in $G F\left(p^{n}\right)$, then $\gamma=\theta^{\frac{p^{n}-1}{p^{l m}-1}}$ is a primitive element in $G F\left(p^{\ell m}\right)$. Therefore, the solutions of equation $(2.4)$ are of the form:

$\gamma^{r^{\prime}}+\frac{\delta}{1-a}$ where $r^{\prime} \equiv \frac{p^{l m}-1}{p^{m}-1} \cdot \frac{k_{a}^{\prime}}{p-1}\left(\bmod \frac{p^{l m}-1}{p^{m}-1}\right)$ and $a=\gamma^{\frac{p^{\ell m}-1}{p-1} k_{a}^{\prime}}$. Since

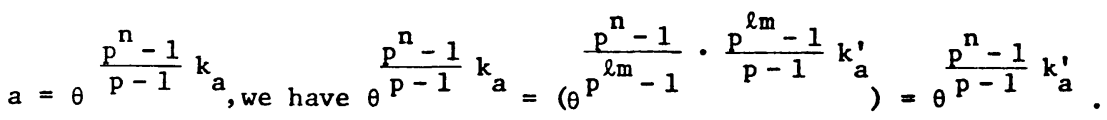

So, $k_{a}=k_{a}^{\prime}$. Therefore, the solutions of $(2.4)$ over $G F\left(p^{n}\right)$ are of the form $\theta^{r}+\frac{\delta}{1-a}$, where

$$
r=\frac{p^{n}-1}{p^{l m}-1} \cdot r^{\prime} \equiv \frac{p^{n}-1}{p^{m}-1} \cdot \frac{k_{a}}{p-1}\left(\bmod \frac{p^{n}-1}{p^{m}-1}\right) .
$$


LEMMA 2.6. Equation (2.4) has $\mathrm{p}^{\mathrm{m}}$ solutions in $\mathrm{GF}\left(\mathrm{p}^{\mathrm{n}}\right)$.

PROOF. Corollary 2.1 insures that equation (2.4) has solution in GF( $\mathrm{p}^{\ell \mathrm{m}}$ ). From the previous note, Theorem 2.1 and Theorem 2.2 we have $\alpha_{0}=\theta_{p^{m}-1}^{\frac{p^{n}-1}{p-1}}$ and $\alpha_{0}+\frac{\delta}{1-a}$ is a solution and if $\alpha \in H=\left\{\alpha_{0} \cdot \beta+\frac{\delta}{1-a} \mid \beta \in G F\left(p^{m}\right)\right\}$, then $\alpha$ is a solution of $(2.4)$. It is clear that $H$ has $\mathrm{p}^{\mathrm{m}}$ elements. $O(a) \mid \frac{n}{m}$, there exists a fundamental set in $G F\left(p^{n}\right)$ with index $m$ and coefficient a. PROOF. By Lemma 2.6, $\quad x^{p^{m}}=$ ax has solutions in $G F\left(p^{n}\right)$. If $\theta$ is a $\frac{p^{n}-1}{p^{m}-1} \cdot k$

primitive element in $G F\left(p^{n}\right)$ then $\alpha=\theta_{e^{m}-1} a$ is a solution. We claim that $\alpha$ has an index $m$ with coefficient a. To prove this, we assume $\alpha$ has an index $\ell<m$ with coefficient $b$, therefore by Theorem 1.4 and Theorem 2.2 we conclude that $\alpha$ is a solution of $x^{p^{l}}=b x$ and $1 t$ is of the form $\theta^{s}$ where $s \equiv \frac{p^{n}-1}{p^{l}-1} \cdot \frac{k b}{p-1} \bmod \left(\frac{p^{n}-1}{p^{l}-1}\right)$. So, there exists $j$ where $j \geq 0$ such that:

$$
\frac{p^{n}-1}{p^{l}-1} \cdot \frac{k_{b}}{p-1}+j \frac{p^{n}-1}{p^{l}-1}=\frac{p^{n}-1}{p^{m}-1} \cdot \frac{k_{a}}{p-1}
$$

then $0 \leq j=\frac{1}{p-1}\left[\frac{k_{a}}{p^{m}-1 / p^{\ell}-1}-k_{b}\right]$.

Since $\frac{p^{m}-1}{p^{l}-1}>p, k_{a}<p-1$ and $1 \leq k_{b}$ we will have

$$
0 \leq j<\frac{1}{p-1}\left[\frac{p-1}{p}-1\right]<0
$$

and this is a contradiction.

Hence $A=A_{\alpha}$ is a fundamental set with index m and coefficient $a$.

COROLLARY 2.2. The minimum subfield which contains all the solutions of equation (2.4) is $\mathrm{GF}\left(\mathrm{p}^{\mathrm{lm}}\right)$.

PROOF. The proof is a direct application of Theorem 1.3 and Theorem 2.4.

We will now review some known facts about the solutions of $x^{p^{m}}=x+b$ in the field $G F\left(p^{n}\right)$.

Let $x^{p^{m}}=x+b$, where $b \in G F\left(p^{n}\right)$

and $d=g \cdot c \cdot d \cdot(m, n)$ and $r=\frac{n}{d}$. The following theorems were given in [1].

LEMMA 2.7. If $x_{0}$ is a solution of (2.5) in $G F\left(p^{n}\right)$, then for every

$j=1,2, \ldots, p^{d}-1,\left(x_{0}+\theta^{\frac{p^{n}-1}{d}-1}\right)$ is a solution of (2.5) where $\theta$ is a primitive element in $G F\left(p^{n}\right)$. 
PROOF.

$$
\begin{aligned}
& \left(x_{0}+{ }_{\theta}^{p^{d}-1}\right)^{p^{n}-1}=x_{0}^{p^{m}}+\left(\theta^{p^{m}}\right)^{\frac{p^{n}-1}{d}-1} \\
& =\left(x_{0}+b\right)+\theta^{\frac{p^{n}-1}{d}-1} j=\left(x_{0}+\theta^{\frac{p^{n}-1}{d}-1} j\right)+b
\end{aligned}
$$

THEOREM 2.5. The number of solutions of (2.5) in $G F\left(p^{n}\right)$ is either 0 or $p^{d}$. THEOREM 2.6. Equation (2.5) has solutions in $G F\left(p^{n}\right)$ if and only if

$\sum_{\ell=0}^{r-1} b^{p^{\ell d}}=0$.

If we assume $m$ divides $n$ and $b \in z_{p}^{*}$ in the equation $x^{p^{m}}=x+b$ then we can conclude the following:

(a) $d=m=g \cdot c \cdot d(m \cdot n)$,

(b) for every $b \in z_{p}^{*}$, we have

$$
\sum_{\ell=0}^{\frac{n}{m}-1} p^{\ell m}=\sum_{\ell=0}^{\frac{n}{m}-1} b=\frac{n}{m} b=0
$$

if and only if $\mathrm{p}$ divides $\frac{\mathrm{n}}{\mathrm{m}}$.

Now, we can restate the following theorems:

(a) Theorem 2.7. Equation $(2.6)$ has solution in $G F\left(p^{n}\right)$ if and only if $p$ divides $\frac{\mathrm{n}}{\mathrm{m}}$.

(b) Theorem 2.8. If equation (2.6) has a solution in $G F\left(p^{n}\right)$, it has $p^{m}$

$$
\begin{aligned}
& \text { solutions, also if } x_{0} \text { is a solution then } x_{0}+{ }_{\theta}^{p^{m}-1}, \\
& j=0,1, \ldots, p^{m}-1 \text { is a solution where } \theta \text { is primitive in } G F\left(p^{n}\right) \text {. }
\end{aligned}
$$

THEOREM 2.9. If $(2.6)$ has a solution in $G F\left(p^{n}\right)$, then the minimum subfield that contain all the solutions is $G F\left(p^{\mathrm{pm}}\right)$.

PROOF. Since equation (2.6) has a solution and $m$ divides $n$ then by Theorem 2.7 p divides $\frac{n}{m}$, therefore $G F\left(p^{p m}\right)$ is a subfield of $G F\left(p^{n}\right)$. If a is a solution of (2.6), then we have $\alpha^{p^{m}}=\alpha+b$, which implies $\alpha^{p^{p m}}=\alpha+p b=\alpha$ so $\alpha \in G F\left(p^{p m}\right)$ and $\alpha \notin \mathrm{GF}\left(\mathrm{p}^{\mathrm{m}}\right)[\mathrm{b} \neq 0]$.

Let $G F\left(p^{l}\right)$ be the minimum subfield which contains all the solutions of (2.6) therefore

$$
G F\left(p^{l}\right) \subset G F\left(p^{p m}\right)
$$

and $\ell \neq m$. This implies $\ell / p m$. But since equation (2.6) has $\mathrm{p}^{\mathrm{m}}$ solutions in $G F\left(\mathrm{p}^{\ell}\right)$ and by Theorem 2.5 and Theorem 2.6, $\mathrm{g} \cdot \mathrm{c} \cdot \mathrm{d}(\mathrm{m}, \ell)=\mathrm{m}$. Hence, $\mathrm{m}|\ell| \mathrm{pm}$.

Therefore, $\mathrm{pm}=\ell$.

LEMMA 2.8. If $\alpha$ is a solution of (2.6) in $G F\left(p^{n}\right)$ where $\alpha$ has an index $s$ with coefficient a then $s$ divides $m$ and $a=1$. 
PROOF. Theorem 1.4 implies $\mathrm{s} / \mathrm{m}$. Let $\frac{\mathrm{m}}{\mathrm{s}}=\mathrm{r}$. Assumes $\mathrm{a} \neq 1$ and $\alpha^{p^{s}}=a \alpha+\delta$ for some $\delta \in Z_{p}$, therefore $\alpha^{p^{m}}=\alpha^{p^{r s}}=a^{r} \alpha+\left(a^{r-1}+a^{r-2}+\ldots+1\right) \delta=$ $a+b$. Hence $a^{r}=1$ and $a^{r-1}+a^{r-2}+\ldots+1=0$ which implies $b=0$, but this contradicts the condition $b \neq 0$ of equation (2.6).

COROLLARY 2.3. In $G F\left(p^{n}\right)$, for every $m$ divides $n$ and $p$ divides $\frac{n}{m}$, there exist $(\mathrm{p}-1) \mathrm{p}^{\mathrm{m}}$ elements $\alpha$, where $\alpha \in \mathrm{A}_{\alpha}$, such that $\mathrm{A}_{\alpha}^{\mathrm{p}^{\mathrm{m}}}=\mathrm{A}_{\alpha}$ and $\mathrm{A}_{\alpha} \not f \mathrm{GF}\left(\mathrm{p}^{\mathrm{m}}\right)$.

PROOF. Equation (2.6) has $p^{m}$ solutions over $G F\left(p^{n}\right)$ for fixed $b \in z_{p}^{*}$ and there are $p-1$ different values for $b$.

COROLLARY 2.4. In $G F\left(p^{n}\right)$, if $m$ is a prime, then all solutions of (2.6) have index $m$ with coefficient $a=1$.

PROOF. This is a direct consequence of Lemma 2.8 .

THEOREM 2.10. In equation (2.6) if $\mathrm{p}$ divides $\frac{\mathrm{n}}{\mathrm{m}}$, then there exists an $\alpha$ where $\alpha$ is a solution of (2.6) and $\alpha$ has an index $m$ with coefficient 1 .

PROOF. Since $p \mid \frac{n}{m}$, then (2.6) has $p^{m}$ solutions. Also by Lemma 2.8 , if $\alpha$ has an index $s$ with coefficient $a=1$ where $s / m$, then $\alpha$ satisfies $x^{p^{8}}=x+\delta \quad(2.7)$ where $\delta=\frac{m}{s}(b)$, hence $s \mid m$ and $p \mid \frac{n}{m}$. By Theorem 2.7 and Theorem 2.8, equation (2.7) has $\mathrm{p}$ solutions. Let $\left\{\mathrm{s}_{1}, \mathrm{~s}_{2}, \ldots, \mathrm{s}_{\ell}\right\}$ be the set of all the indices of the solutions of (2.6) such that $1 \leq s_{i}<s_{j}<m$ for every $1<j \leq \ell$. Since $s_{\ell} \leq\left[\frac{\mathrm{m}}{2}\right]$ where $\left[\frac{\mathrm{m}}{2}\right]$ is the greatest integer less than or equal to $\frac{\mathrm{m}}{2}$, we have

$$
\begin{aligned}
& \mathrm{p}^{\mathrm{m}} \leq \mathrm{p}^{\mathrm{s}_{1}}+\mathrm{p}^{\mathrm{s}_{2}}+\ldots+\mathrm{p}^{\mathrm{s}_{\ell}} \leq \mathrm{p}+\mathrm{p}^{2}+\ldots \mathrm{p}^{\left[\frac{\mathrm{m}}{2}\right]} \\
& =\frac{p\left[\frac{m}{2}\right]+1}{p-1}
\end{aligned}
$$

But since $\frac{p^{k+1}-p}{p-1}<p^{2 k}$ for $k \geq 1$ and $\frac{p^{k+1}-p}{p-1}<p^{2 k+1}$, we have

$$
p^{m} \leq \frac{p^{\left[\frac{m}{2}\right]+1}-p}{p-1}<p^{m}
$$

and this is a contradiction.

COROLLARY 2.5. In $G F\left(p^{n}\right)$, for every $m$ divides $n$, and $p$ divides $\frac{n}{m}$, there exists a fundamental set with index $m$ and coefficient $a=1$.

3. THE TOTAL NUMBER OF FUNDAMENTAL CLASSES IN SOME FINITE FIELDS.

In this section we will investigate the total number of fundamental classes for some special finite fields. From the previous study we conclude that if $p \mid \frac{n}{m}$, there exists a fundamental set with index $m$ and coefficient 1 in $G F\left(p^{n}\right)$.

In $G F\left(p^{n}\right)$, for every $m$ divides $n$, and every $a \in z_{p}^{*}$, $a \neq 1$ where $a^{\frac{n}{m}}=1$ there exists a fundamental set with index $m$ and coefficient $a$. Since $a^{\frac{n}{m}}=1$ then the $g \cdot c \cdot d\left(\frac{n}{m}, p-1\right) \neq 1$.

If $A$ is a fundamental set with index $m$ then $\bar{A}$ has $p(p-1)$ m elements. 
In this section we will use the following notations:

(a) $0\left(p^{n}\right)=$ Number of fundamental classes in $G F\left(p^{n}\right)$.

(b) $\Delta\left(p^{n}\right)=$ Number of fundamental classes in $G F\left(p^{n}\right)$ but not in any proper subfield of $G F\left(p^{n}\right)$.

(c) $\lambda\left(p^{n}, m, a\right)=$ Number of elements in GF( $\left.p^{n}\right)$ with index $m$ and coefficient a and none of these elements belonging to any proper subfield of $G F\left(p^{n}\right)$.

(d) $E(m, a)=\left\{x^{p^{m}}=a x+\delta ; \delta \in z_{p}, a \in z_{p}^{*}\right.$ and $\delta \neq 0$ if $\left.a=1\right\}$.

(e) $\mathrm{SE}(\mathrm{m}, \mathrm{a}, \mathrm{n})$ is the set of all solutions of the equations of $E(m, a)$ in $G F\left(p^{n}\right)$ but not in $z_{p}$.

We shall investigate in the following the number of fundamental classes in $\operatorname{GF}\left(\mathrm{p}^{\mathrm{q}^{\ell}}\right)$, where $\mathrm{q} p-1$, and $\mathrm{q} \neq \mathrm{p}$.

LEMMA 3.1. If $q / p-1$, then $q^{t+1}$ divides $p^{t}[q-1]-1$ for every $t=0,1,2, \ldots$.

PROOF. We will prove this lemma by induction. By Fermat Theorem the lemma is true for $t=0$. Assume it is true for $t=s$ then

$$
\begin{aligned}
& {\left[p^{q^{s+1}[q-1]}-1\right]=\left[\left(p^{q^{s}[q-1]}\right)^{q^{s}}-1\right]} \\
& =\left(p^{q^{s}[q-1]}-1\right)\left[\left(p^{q^{s}[q-1]}\right)^{q^{s}-1}+\left(p^{q^{s}[q-1]}\right)^{q^{s}-2}+\ldots+1\right] \\
& {\left[p^{q^{s}[q-1]}-1\right]\left[\sum_{j=1}^{q}\left(p^{q^{s}[q-1]}\right)^{q^{s-j}}\right] .}
\end{aligned}
$$

Since $p^{q^{s}[q-1]} \equiv 1 \bmod q$, then $\left(p^{q^{s}[q-1]}\right)^{q^{s}-j} \equiv 1(\bmod q)$ for every $j=0,1, \ldots, q^{s}$. So, $\sum_{j=1}^{q^{s}}\left(p^{q^{s}[q-1]}\right)^{q^{s}-j} \equiv q^{s}(\bmod q) \equiv 0(\bmod q)$ which implies $q^{s+2}$ divides $\left[p^{q^{s+1}}[q-1]-1\right]$

LEMMA 3.2. In GF ( $\mathrm{p}^{\mathrm{t+1}}$ ) where $\mathrm{q} / \mathrm{p}-1$ and $q \neq \mathrm{p}$, we have $\Delta\left(p^{t+1}\right)=\frac{p^{q^{t}}\left[p^{q^{t}[q-1]}-1\right]}{p(p-1) q^{t+1}}$.

PROOF. Since $g \cdot c \cdot d(q, p-1)=1$, then for every $a \in z_{p}^{*}$, where $a \neq 1$, $a^{q^{8}} \neq 1$ for every $s \geq 0$, therefore equation (2.6) and equation (2.7) have no solutions in $G F\left(p^{q+1}\right)$ for every $m=q^{h} ; h \geq 0$. Hence every element in $G F\left(p^{t+1}\right)$ but not in $G F\left(p^{q}\right)$ has index $q^{t+1}$ which implies that every fundamental set in $G F\left(p^{t+1}\right.$ ) but not in $G F\left(\mathrm{p}^{\mathrm{t}}\right.$ ) also has index $\mathrm{q}^{\mathrm{t}+1}$. So:

$$
\Delta\left(p^{t+1}\right)=\frac{p^{q^{t+1}}-p^{q^{t}}}{p(p-1) q^{t+1}}=\frac{p^{q^{t}}\left[p^{q^{t}[q-1]}-1\right]}{p(p-1) q^{t+1}}
$$

From Lemma 3.1 and $g \cdot c \cdot d(p-1, q)=1$ we have $\Delta\left(p^{t+1}\right)$ is an integer.

COROLLARY 3.1 In $G F\left(p^{q}\right)$ where $g / p-1, q \neq p$ we have:

$$
O\left(p^{q}\right)=1+\frac{p^{q}-p}{p(p-1) q}=1+\frac{p\left(p^{q-1}-1\right)}{p(p-1) q} \text {. }
$$


Since $o\left(p^{q^{t+1}}\right)=o\left(p^{q^{t}}\right)+\Delta\left(p^{q^{t+1}}\right)$ we conclude that:

$$
\begin{aligned}
o\left(p^{\ell}\right) & =1+\sum_{t=0}^{\ell-1} \Delta\left(p^{q^{t+1}}\right) \\
& =1+\sum_{t=0}^{\ell-1} \frac{p^{q^{t}}\left[p^{q^{t}[q-1]}-1\right]}{p(p-1) q^{t+1}}
\end{aligned}
$$

where $\mathrm{q} / \mathrm{p}-1, \mathrm{q} \neq \mathrm{p}$.

We shall now study the number of fundamental classes in GF ( $\left.p^{s}\right)$.

By Theorem 2.7, Theorem 2.8 and Theorem 2.9, the equation

$x^{p^{p}}=x+b$

where $b \in z_{p}^{*}$ and $t<s$ has $p^{p^{t}}$ solutions and all solutions are included in $G F\left(p^{p^{t+1}}\right)$.

LEMMA 3.3. All the solutions of (3.2) have an index $p^{t}$.

PROOF. Let $\alpha$ be a solution of (3.2) and has index $m$. We have $m=p^{k}$ for some $k<t$ and $\alpha^{p^{p^{k}}}=\alpha+c$ for some $c \in Z_{p}$ which implies $\alpha^{p^{p^{t}}}=\alpha+0=\alpha$. Therefore $\alpha$ is not a solution of (3.2).

COROLLARY 3.2. In GF $\left(p^{p^{t+1}}\right)$ but not in $G F\left(p^{p^{t}}\right)$, there are $(p-1) p^{p^{t}}$ elements with index $p^{p^{t}}$. So $\lambda\left(p^{p^{t+1}}, p^{t}, 1\right)=(p-1) p^{p^{t}}$.

PROOF. For a fixed $b \in z_{p}^{*}$ equation (3.2) has $p^{p^{t}}$ solutions and we have $(p-1)$ elements in $\mathrm{z}_{\mathrm{p}}^{*}$.

COROLlaRY 3.3. If $\alpha \in G F\left(p^{p^{t+1}}\right)$ but $\alpha \notin G F\left(p^{t}\right)$ then $\alpha$ has index $p^{t+1}$ or $p^{t}$, where $t \geq 1$.

COROLLARY 3.4 . For $t \geq 1$;

$$
\begin{aligned}
\Delta\left(p^{p^{t+1}}\right) & =\frac{(p-1) p^{p^{t}}}{p(p-1) p^{t}}+\frac{p^{p^{t+1}}-p^{p^{t}}-(p-1) p^{p^{t}}}{p(p-1) p^{t+1}} \\
& =\frac{p^{p^{t}}}{p^{t+1}}+p^{p^{t}-t-1}\left[\frac{p^{p^{t}(p-1)-1}-1}{(p-1)}\right] \\
& =p^{p^{t}-t-1}\left[\frac{p^{p^{t}(p-1)-1}-1}{(p-1)}+1\right] .
\end{aligned}
$$

In $G F\left(p^{p}\right)$, the equation $x^{p}=x+b$

where $b \in Z_{p}^{*}$ has $p$ solutions and each solution has index $p$. Therefore there are $p(p-1)$ elements in $G F\left(p^{p}\right)-Z_{p}$ with index $p$. This implies:

LEMMA 3.4 .

$$
\begin{aligned}
o\left(p^{p}\right) & =1+\frac{p(p-1)}{p(p-1)}+\frac{p^{p}-p(p-1)-p}{p(p-1) p} \\
& =2+\frac{p^{p-2}-1}{p-1}
\end{aligned}
$$


COROLLARY 3.5 .

$$
O\left(p^{p^{s}}\right)=2+\frac{p^{p-2}-1}{p-1}+\sum_{t=1}^{s-1} \Delta\left(p^{p^{t+1}}\right)
$$

In what follows we will study the number of fundamental classes in $G F\left(p^{p^{s} \cdot q^{\ell}}\right.$ ) where $q / p-1, q \neq p$ and $s, l \geq 1$.

Since $q / p-1$ then for every $m$ divides $p^{s} \cdot q^{\ell}$ and every $a \in z_{p}^{*}$ with $a \neq 1$, we $\frac{p^{s} \cdot q^{\ell}}{m}$

have $a{ }^{m} \neq 1$. So, Theorem 1.3 implies that $\operatorname{SE}\left(m, a, p^{s} q^{\ell}\right)=\phi$ for every $a \neq 1$ and every $\mathrm{m} \mid \mathrm{p}^{\mathrm{s}} \cdot \mathrm{q}^{\ell}$.

By Theorem 2.7, Theorem 2.8 and Theorem 2.9, we conclude the following:

LEMMA 3.5. For every $t$ and $h ; 0 \leq t \leq s-1,0 \leq h \leq \ell, \operatorname{sE}\left(p^{t} q h, 1, p^{h} q^{l}\right)$ has $(p-1) p^{p} \cdot q^{h}$ elements, all of them are contained in the minimum subfield $G F\left(p^{p^{t+1} q^{h}}\right.$ ) and $\operatorname{SE}\left(p^{t} q^{h}, 1, p^{s} q^{l}\right) \cap G F\left(p^{t} \cdot q^{h}\right)=\phi$.

LEMMA 3.6. For every $t$, $h$ such that $0 \leq t \leq s-1,0 \leq h \leq l-1$, $\operatorname{SE}\left(p^{t} q^{h}, 1, p^{s} q^{l}\right)$ is included in $\operatorname{SE}\left(p^{t} q^{h+1}, 1, p^{s} q^{l}\right)$.

PROOF. Let $\alpha \in \operatorname{SE}\left(p^{t} q^{h}, 1, p^{s} q^{l}\right)$. Then $\alpha^{p^{p^{t}} q^{h}}=\alpha+\delta$ for some $\delta \in z_{p}^{*}$, which implies $\alpha^{p^{p^{t} \cdot q^{h}+1}}=\alpha+[q \cdot \delta]$, where [x] is the least nonnegative residue of $x \bmod p$. Since $[q \cdot \delta] \neq 0$ then $\alpha \in \operatorname{SE}\left(p^{t} \cdot q^{h+1}, 1, p^{s} q^{l}\right)$.

It is clear that if $\alpha \in \operatorname{SE}\left(p^{t} q^{h}, 1, p^{s} q^{l}\right)$, then $\alpha$ has an index $p^{t} \cdot q^{r}$ for some $0 \leq \mathrm{r} \leq \mathrm{h}$. Since, $\operatorname{sE}\left(\mathrm{p}^{\mathrm{t}}, 1, \mathrm{p}^{\mathrm{s}} \mathrm{q}^{l}\right) \subset \operatorname{SE}\left(\mathrm{p}^{\mathrm{t}} \mathrm{q}, 1, \mathrm{p}^{\mathrm{s}} \mathrm{q}^{l}\right) \subset \ldots \subset \operatorname{sE}\left(\mathrm{p}^{t} \mathrm{q}^{\mathrm{h}+1}, 1, \mathrm{p}^{\mathrm{s}} \mathrm{q}^{\ell}\right)$ we conclude that

$$
\begin{gathered}
\lambda\left(p^{p^{t+1} \cdot q^{h+1}}, p^{t} q^{h+1}, 1\right)=(p-1) p^{p^{t} \cdot q^{h+1}}-(p-1) p^{p^{t} \cdot q^{h}} \\
=(p-1) p^{p^{t} \cdot q^{h}}\left[p^{p^{t} \cdot q^{h}[q-1]}-1\right] .
\end{gathered}
$$

Therefore, in GF ( $\left.p^{p^{t+1}} q^{h+1}\right)$ there are

$$
\frac{(p-1) p^{p^{t} \cdot q^{h}\left[p^{p^{t} \cdot q^{h}[q-1]}-1\right]}}{p(p-1) p^{t} \cdot q^{h+1}}=\frac{\left.p^{p^{t} \cdot q^{h}\left[p^{p} \cdot q^{h}[q-1]\right.}-1\right]}{p^{t+1} \cdot q^{h+1}}
$$

fundamental classes with index $p^{t} \cdot q^{h+1}$. From Lemma 3.1 and the fact that $p^{t} \geq t+1$, the number given in (3.8) is an integer.

Since any proper subfield of $G F\left(p^{p^{t+1} \cdot q^{h+1}}\right)$ is a subfield of $G F\left(p^{\left.p^{t} \cdot q^{h+1}\right)}\right.$ or $G F\left(p^{p^{t+1} \cdot q^{h}}\right)$. So, in $G F\left(p^{p^{t+1} \cdot q^{h+1}}\right)$, the number of fundamental classes with index $p^{t+1} \cdot q^{h+1}$ is equal to

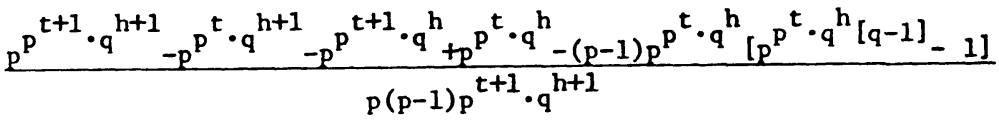

$$
\begin{aligned}
& =\frac{p^{p^{t+1} \cdot q^{h}}\left[p^{p^{t+1} \cdot q^{h}[q-1]}-1\right]-p^{p^{t} \cdot q^{h}}\left[p^{p^{t} \cdot q^{h}[q-1]}-1\right] \cdot p}{p(p-1) p^{t+1} \cdot q^{h+1}}
\end{aligned}
$$


THEOREM 3.11. In GF $\left(\mathrm{p}^{\mathrm{p}^{\mathrm{s}} \cdot \mathrm{q}^{\ell}}\right)$,

$$
\Delta\left(p^{p^{t+1} \cdot q^{n+1}}\right)=(3.8)+(3.9) .
$$

From it follows that

$$
\begin{aligned}
o\left(p^{p^{t+1} \cdot q^{h+1}}\right) & =\Delta\left(p^{p^{t+1} \cdot q^{h+1}}\right)+o\left(p^{p^{t+1} \cdot q^{h}}\right) \\
& +o\left(p^{p^{t} \cdot q^{h+1}}\right)-o\left(p^{p^{t} \cdot q^{h}}\right) .
\end{aligned}
$$

We now study the general case, i.e. the number of fundamental classes in $G F\left(p^{n}\right)$. First, let $n=q_{1}{ }^{\ell} \cdot q_{2}{ }^{\ell_{2}} \ldots q_{r}{ }_{r}^{l_{r}}$ such that $\ell_{i} \neq 0 g_{i} / p-1$ and $q_{i} \neq p$ for every $i=1,2, \ldots, r$.

Let $N(s, p)$ be the number of elements in $G F\left(p^{s}\right)$ but not in any proper subfield of $\mathrm{GF}\left(\mathrm{p}^{\mathrm{s}}\right)$.

THEOREM 3.12. The number $N(s, p)=\sum_{i \cdot j=s} \mu(i) q^{j}$ where $\mu$ is the Mobius functions.
[2]). (See [2]).

Since for every $m$ divides $n$ and every $a \in z_{p}^{*}$, the set $\operatorname{SE}(m, a, n)=\phi$. It implies that if $\alpha \in G F\left(p^{k}\right)$ where $G F\left(p^{k}\right)$ is a subfield of $G F\left(p^{n}\right)$ and $\alpha$ doesn't belong to any proper subfield of $G F\left(p^{k}\right)$, then $\alpha$ has an index $p^{k}$. So, we have the following

THEOREM 3.13. If $G F\left(p^{k}\right) \subseteq G F\left(p^{n}\right)$ then $\Delta\left(p^{k}\right)=\frac{N(k, p)}{p(p-1) p^{k}}$.

Now let $n=p^{k} q_{1}{ }^{\ell} \cdot q_{2}{ }^{\ell} \cdot \ldots q_{s}^{\ell_{s}}$ such that $k \neq 0, q_{i} \neq p_{i}, l_{i} \neq 0$ and $q_{i} / p-1$ for every $i=1,2, \ldots, s$.

LEMMA 3.7. For every $m=p^{t} \cdot q_{1}^{t}{ }^{1} \cdot q_{2}{ }^{t} \cdot \ldots \cdot q_{s}^{t} s$ where $0 \leq t \leq k-1$, $0 \leq t_{i} \leq \ell_{i}, i=1,2, \ldots, s$ then $\operatorname{SE}(m, 1, n)$ has $(p-1) p^{m}$ elements, all of them are contained in the minimum subfield $G F\left(p^{p m}\right)$ and $\operatorname{SE}(m, 1, n) \cap G F\left(p^{m}\right)=\phi$.

Since $\operatorname{SE}(\mathrm{m}, 1, \mathrm{n}) \subset \mathrm{GF}\left(\mathrm{p}^{\mathrm{pm}}\right)$, we have $\operatorname{SE}(\mathrm{m}, 1, \mathrm{n})=\operatorname{SE}(\mathrm{m}, 1, \mathrm{pm})$.

LEMMA 3.8. For every $m=p^{t} \cdot q_{1}{ }^{t} \cdot \ldots \cdot q_{s}^{t} s$ where $0 \leq t \leq k-1,0 \leq t \leq l_{i}$ for $i=1,2, \ldots, s$ and for some $t_{r} ; t_{r} \leq \ell_{r}-1, S E(m, 1, n)$ is a proper subset of $\mathrm{SE}(\mathrm{mq}, 1, \mathrm{n})$.

LEMMA 3.9. If $\mathrm{p}^{k}$ divides $m$, and $m$ divides $n$ then $S E(m, 1, n)=\phi$.

COROLLARY 3.6. If $\operatorname{SE}\left(\mathrm{mq}_{\mathrm{r}_{1}}, 1, \mathrm{n}\right) \neq \phi$ and $\operatorname{SE}\left(\mathrm{mq}_{\mathrm{r}_{2}}, 1 ; n\right) \neq \phi$ for some $\mathrm{r}_{1} \neq \mathrm{r}_{2}$ then $\operatorname{SE}\left(\mathrm{mq}_{\mathrm{r}_{1}}, 1, \mathrm{n}\right) \neq \operatorname{SE}\left(\mathrm{mq}_{\mathrm{r}_{2}}, 1, \mathrm{n}\right)$.

The proof is an immediate application of Lemma 3.7 .

COROLLARY 3.7. If $\mathrm{p}^{\mathrm{t}}$ divides $\mathrm{m}$ but not $\mathrm{r}$, then $\operatorname{SE}(\mathrm{m}, 1, \mathrm{n}) \cap \mathrm{SE}(\mathrm{r}, 1, \mathrm{n})=\phi$.

The proof is a direct application of Lemma 3.7 .

COROLLARY 3.8. If for some $0 \leq t \leq k-1 \mathrm{p}^{\mathrm{t}} / \mathrm{m}$ and $\mathrm{p}^{\mathrm{t}} / \mathrm{r}$ but $\mathrm{p}^{\mathrm{t}+1} / \mathrm{m}$ and $\mathrm{p}^{\mathrm{t}+1}$ /r then

$$
\operatorname{SE}(m, 1, n) \cap \operatorname{SE}(r, 1, n)=\operatorname{SE}((m, r), 1, n)
$$

where $(m, r)=g \cdot c \cdot d(m, r)$.

The proof is a direct application of Lemma 3.7, Corollary 3.6 and 
Corollary 3.8 .

We know from Theorem 2.10 if $m$ divides $n$ and $\operatorname{SE}(m, 1, n) \neq \phi$ then there is an $\alpha \in \operatorname{SE}(m, 1, n)$ such that $\alpha$ has an index $m$. We want to find the number of elements in $\mathrm{SE}(\mathrm{m}, \mathrm{l}, \mathrm{n})$ with index $\mathrm{m}$. Let $\mathrm{m}=\mathrm{p}^{\mathrm{t}} \cdot \mathrm{q}_{1_{1}}{ }^{\mathrm{t}}{ }_{1} \cdot \mathrm{q}_{1_{2}}{ }^{\mathrm{t}} \mathrm{l}_{2} \cdot \ldots \cdot \mathrm{q}_{1_{\mathrm{h}}}{ }^{\mathrm{t}}$ and $\mathrm{m}$ divides $\mathrm{n}$ where $0 \leq t \leq k-1, t_{1 r} \neq 0$ for $r=1, \ldots, h$ and $h<s$. If we rearrange the $q^{\prime} s$ in the factorization of $n$ such that $q_{1 r}=q_{r}$ for $r=1,2, \ldots, h$, then $\operatorname{sE}\left(\frac{m}{q_{i}}, 1, n\right) \subset \operatorname{sE}(m, 1, n)$ for every $i=1,2, \ldots, h$, and $\bigcap_{i=1}^{h} \operatorname{sE}\left(\frac{m}{q_{i}}, 1, n\right)=\operatorname{sE}\left(\frac{m}{q_{1} q_{2} \cdots q_{h}}, 1, n\right)$.

We shall use the following notations for the remaining of this section:

$$
\mathrm{m}=\mathrm{p}^{\mathrm{t}} \cdot \mathrm{q}_{1}^{\mathrm{t}}{ }_{1} \cdot \mathrm{q}_{2}{ }^{\mathrm{t}} \cdot \ldots \cdot \mathrm{q}_{\mathrm{h}}^{\mathrm{t}}{ }^{\mathrm{h}} ; 1 \leq \mathrm{t}_{i}
$$

and

$$
\begin{aligned}
& \mathrm{m}_{1}=\sum_{i=1}^{\mathrm{h}} \frac{\mathrm{m}}{\mathrm{q}_{i}} \\
& m_{2}=\sum_{i, j=1}^{h} \frac{m}{q_{i}^{q} q_{j}} \\
& m_{r}=\sum_{i_{j}=1}^{h} \frac{m}{q_{i_{1}} \cdot q_{i_{2}} \cdot \ldots \cdot q_{i_{r}}} \\
& i<j \\
& 1 \leq i_{1}<i_{2}<\ldots<i_{r} \leq h \text {, and } \\
& \prod_{j=1}^{r} q_{i j} \neq \prod_{j=1}^{r} q_{\ell} \text { for } i \neq \ell \text {. }
\end{aligned}
$$

If $r>h$ then we define $m_{r}=0$. Therefore $m_{h}=p^{t} \cdot q_{1}{ }^{t}{ }^{-1} \cdot q_{2}{ }^{t} \cdot \ldots \cdot q_{h}{ }^{t} \cdot$. From previous Lemmas, we conclude the following.

If $\operatorname{R}[\operatorname{SE}(m, 1, n)]$ is the number of elements in $\operatorname{SE}(m, 1, n)$ with index $m$ then

$$
\mathrm{R}[\mathrm{SE}(\mathrm{m}, 1, \mathrm{n})]=\mathrm{m}-\mathrm{m}_{1}+\mathrm{m}_{2}-\mathrm{m}_{3}+\ldots+(-1)^{\mathrm{r}_{\mathrm{m}_{\mathrm{r}}}}+\ldots+(-1)^{\mathrm{h}_{\mathrm{m}}} \text {. }
$$

Also, if $B \in G F\left(p^{p m}\right)$ and not in any proper subfield of $G F\left(p^{p m}\right)$, then $B$ has index $\mathrm{m}$ or $\mathrm{pm}$. Hence we have the following.

THEOREM 3.14 .

$$
\Delta\left(p^{p m}\right)=\frac{R[S E(m, 1, p m)]}{p(p-1) m}-\frac{N(p m, p)-R[S E(m, 1, p m)]}{p(p-1) p m} .
$$

To determine the number of equivalence classes in $G F\left(p^{q}\right)$ where $q / p-1$, we need to study $\operatorname{SE}\left(\mathrm{m}, \mathrm{a}, \mathrm{q}^{\mathrm{s}}\right)$ for all $\mathrm{m}$ dividing $\mathrm{q}^{\mathrm{s}}$ and $\mathrm{a} \in \mathrm{z}_{\mathrm{p}}^{*}$. From Theorem 2.7 we have $\operatorname{SE}\left(M, 1, q^{S}\right)=\phi$ for every $m / q^{S}$. Also we know that for every $m \mid q^{s}$ and every a $\neq 1$, $a \in z_{p}^{*}$ if $a^{q^{s} / m}=1$ then $S E\left(m, a, q^{s}\right)$ has $P\left(p^{m}-1\right)$ elements. One question we will try to answer first is that for given $m$, is there an $a \in z_{p}^{*}$, a $\neq 1$ such that $a^{s} / m=1$ and then how many such $a^{\prime} s$ in $z_{p}^{*}$ can one find? Another question is for fixed $i$ and $a$, how many $m^{\prime} ' s, a^{\prime}$ 's are there such that $\operatorname{SE}\left(m^{\prime}, a^{\prime}, q^{s}\right) \subset E\left(m, a, q^{s}\right)$. order $q^{\mathrm{v}}$.

LEMMA 3.10. In $z_{p}$, if $q^{v}$ divides $(p-1)$ then there are $q^{v \cdot 1}(\varphi-1)$ elements of

PROOF. Let $b$ be primitive element in $z_{p}$. Assume for some $k$; $] \leq k \leq p-1$, $b^{k}$ is a solution $x^{q}=1$. This implies $k \cdot q \equiv 0 \bmod (p-1)$. So, $k=t \cdot \frac{\bar{p}-1}{q}$ for some 
$t=1,2, \ldots, q-1$. But also if $r=\ell \frac{p-1}{q}$ for every $\ell=1,2, \ldots, q-1$ we have $\left(b^{r}\right)^{q}=b^{l(p-1)}=1$ and $b^{r} \neq 1$ which implies that in $z_{p}$ we have $(q-1)$ elements of order q. Let $p-1=q^{\ell} \cdot h$ where the $g \cdot c \cdot d(p-1, h)=1$, then for every $r$ such that $r \equiv 0 \bmod \left(q^{\ell-v} \cdot h\right)$ and $0 \leq r<q^{v}$ we have $b^{r}$ is a solution of $x^{q^{v}}=1$, which implies that in $z_{p}$, there are $q^{v}-1$ elements such that $x^{q^{v}}=1$ and $x \neq 1$. For $v=1$, $v=2$ we will have $q^{2}-1-(q-1)=q(q-1)$ elements of order $q^{2}$. The same for $v=t-1, v=t$ we will have $q^{t}-1-\left(q^{t-1}-1\right)=q^{t-1}(q-1)$ elements of order $q^{t}$.

LEMMA 3.11. In $z_{p}$, if for a fixed $b$ where $O(b)=q^{\mu}$ and $1 \leq \mu<\ell$ there exists an a of order $q^{v}$ where $l \geq v>\mu$ and a satisfies $x^{q^{v-\mu}}=b$

then there are $q^{v-\mu}$ distinct elements in $z_{p}^{*}$ of order $q^{v}$ satisfying (3.13)

PROOF. Note that equation (3.13) has no repeated roots. If a is a solution, we claim that $a, a^{q^{\mu}+1}, a^{2 q^{\mu}+1}, \ldots, a^{\left(q^{v-\mu}-1\right) \cdot q^{\mu}-1}$ are distinct solutions of (3.13). To prove our claim, first since $0(a)=q^{v}$ then $a^{r_{i} q^{\mu}+1}$ for $r_{i}=1,2, \ldots, q^{v-\mu}-1$ are distinct clunents in $z_{p}$. Also $\left(a^{r^{q^{\mu}}+1}\right)^{v-\mu}=a^{r \cdot q^{v}} \cdot a^{q^{v-\mu}}=1 \cdot b=b$. Since $g \cdot c \cdot d\left(r q^{\mu}+1, q\right)=1$ and $O(a)=q^{v}$, we iave $O\left(a^{r q^{\mu}+1}\right)=q^{v}$.

THEOREM 3.15. If $b \in z_{p}$ uch that $O(b)=q^{\mu} ; 0<\mu<\ell$, then for every $v$, $\ell \geq v>\mu$, there are $q^{v-\mu}$ ele phents of ortur $q^{v}$ and satisfying (3.13).

PROOF. In $z_{p}$ we have $q^{v-1}(q-1)$ elements of order $q^{v}$. Let $c \in z_{p}$ and $O(c)=q^{v}$ and let $c^{q^{v-\mu}}=d$ so $d \neq 1$ and $d^{q^{\mu}}=1$. Furthermore, the order of $d$ is $q^{\mu}$. By Lema 3.11, we have $q^{v-\mu}$ elements satisfying $x^{q^{v-\mu}}=d$ for each $d$. But there are $\frac{q^{v-1}(q-1)}{q^{n-\mu}}=q^{\mu-1}(q-1)$ distinct $d^{\prime} s$ of order $q^{\mu}$ for which the above equation is solvable and that is exactly the total number of elements in $z_{p}$ having order $q^{\mu}$. LEMMA 3.12. If $q$ divides $p-1$, then $q^{t}$ divides $p^{q^{t}}-1$ for every $t>0$. PROOF. By induction.

In $G F\left(p^{q}\right)$ where $q$ divides $p-1$, the set $\operatorname{SE}(1, a, q) \neq \phi$ if and only if $O(a)=q$. Since there are $(q-1)$ elements of order $q$ and for a fixed b with $0(b)=q$, the set $\operatorname{SE}(1, b, q)$ has $p(p-1)$ elements, therefore $G F\left(p^{q}\right)$ has $\frac{p(p-1)(q-1)}{p(p-1)}=(q-1)$ fundamental classes with index 1 . So we conclude:

$$
\begin{aligned}
o\left(p^{q}\right) & =(q-1)+\frac{p^{q}-p-p(p-1)(q-1)}{p(p-1) \cdot q}+1 \\
& =(q-1)+\frac{\left(p q^{q-1}-1\right)+(p-1)}{(p-1) q} \\
& =(q-1)+\frac{p^{q-2}+p^{q-3}+\ldots+1+1}{q}
\end{aligned}
$$

Since $P \equiv 1 \bmod q$.

$$
1+\sum_{r=2}^{q} p^{q-r} \equiv 1+(q-1) \bmod q \equiv 0 \bmod q .
$$

So, (3.14) is an integer.

THEOREM 3.16. In $\operatorname{GF}\left(\mathrm{p}^{\mathrm{q}}\right)$ where $q$ divides $(\mathrm{p}-1)$, if $O(a)=q^{v}$, then for every $t$, where $0 \leq t \leq s-v$ we have $\operatorname{SE}\left(q^{t}, a, q^{s}\right)=\operatorname{SE}\left(q^{t}, a, q^{t+v}\right) \neq \phi$ and $\operatorname{SE}\left(q^{t}, a, q^{v+\bar{t}}\right) \cap \operatorname{SE}\left(q^{t}, a, q^{v+t=1}\right)=\phi$. 
PROOF. By Corollary 2.1 we have

$$
\operatorname{SE}\left(q^{t}, a, q^{s}\right)=\operatorname{SE}\left(q^{t}, a, q^{v+t}\right) \neq \phi .
$$

Theorem 2.4 and Corollary 2.2 will imply that $x^{p^{q}}=a x+b ; b \in z_{p}$ has $a$ solution $\alpha_{0}$ with index $m=q^{t}$ and $\alpha_{0} \notin G F\left(p^{t+v-1}\right)$. By Theorem 2.1 the solution set of this equation is $H=\left\{\alpha_{0} \cdot \beta+\frac{b}{1-a} / \beta \in G F\left(p^{q}\right)\right\}$ and it is clear that $H \cap G F\left(p^{q^{t+v-1}}\right)=\left\{\frac{b}{1-a}\right\}$.

NOTE: This theorem is not true in general. It is possible that in some cases the minimum subfield that contains all the solutions of $x^{p^{m}}=a x+\delta$ has a proper subfield which contains some of these solutions.

From Theorem 3.16 we conclude that in $\operatorname{CF}\left(\mathrm{p}^{\mathrm{s}}\right)$, where $q^{!} \mathrm{p}-1, \operatorname{SE}\left(\mathrm{q}^{t^{\prime}}, \mathrm{b}, \mathrm{q}^{\mathrm{s}}\right)$ is a

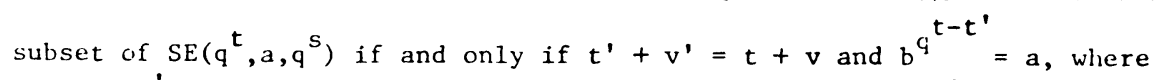
$O(b)=q^{v^{\prime}}, O(a)=q^{v}, t^{\prime} \leq t \leq s-v$. Also for a fixed $a \in z_{p}^{*}$ with order $q^{v}$ where $v>0$, Theorem 3.15 insures the existence of exactly q elements " $b$ " in $z_{p}$ such that $O(b)=q^{v+1}$ and $b^{q}=a$. Hence, for a fixed a with order $q^{v}$ and fixed $t$; $t \leq s-v$ there are $q$ elements $b$ in $z_{p}$ such that:

$$
\operatorname{SE}\left(q^{t-1}, b, q^{s}\right) \subset \operatorname{SE}\left(q^{t}, a, q^{s}\right)
$$

Therefore, if we start with $a_{0} \in z_{p}^{*}$ such that $0\left(a_{0}\right)=q^{v}$, then there are $a_{1}, \ldots, a_{n} \in z_{p}^{*}$ where $0\left(a_{i}\right)^{\prime}=q^{v+i}$ and $\left(a_{i+1}\right)^{q}=a_{i}$. So, we have $\operatorname{SE}\left(q^{t-(i+1)}, a_{i+1}, q^{s}\right) \subset \operatorname{SE}\left(q^{t-i}, a_{i}, q^{s}\right)$ for every $i=0,1,2, \ldots, n-1$ where $t-n \geq 0$ and $\mathrm{q}^{\mathrm{v}+\mathrm{n}} \mid(\mathrm{p}-1)$.

Let $\mathrm{p}-1=\mathrm{q}^{\ell} \cdot \mathrm{h}$ where $\mathrm{g} \cdot \mathrm{c} \cdot \mathrm{d}(\mathrm{h}, \mathrm{q})=1$. Then for every $a \in z_{\mathrm{p}}^{*} \mathrm{a} \neq 1$ and every $t \geq 1$ such that $0(a)=q^{v}, 1 \leq v<\ell$ and $t+v \leq s$ we have the following Lemma.

LEMMA 3.13. In $S E\left(q^{t}, a, q^{s}\right)$, there are exactly $\left(p^{q^{t}}-1\right) p-\left(p^{q^{t-1}}-1\right) p \cdot g$ eloments with index $q^{t}$ and coefficient $a$.

From this lemma, we conclude that in $G F\left(p^{t+v}\right)$ there are

$$
\frac{\left(p^{q^{t}}-1\right)-\left(p^{\left.q^{t-1}-1\right) \cdot q}\right.}{(p-1) \cdot q^{t}} \cdot q^{v-1}(q-1)
$$

fundamental classes and each class has an index $q^{t}$ and a coefficient with order $q^{v}$. Lemma 3.12 insures that (3.15) has an integer value. We w111 use the notation:

$$
\Gamma(m, t, v)=(3.15) \text {, where } m=t+v, 1 \leq v<\ell \text { and } t \geq 1 \text {. }
$$

It is clear now that in $G F\left(\mathrm{p}^{\mathrm{q}}\right)$ where $s \geq 1$, we have

$$
\frac{\left(p^{q^{s}}-p^{q^{s-1}}\right)-p\left(p^{q^{s-1}}-1\right)(q-1)}{p(p-1) q^{s}}
$$


fundamental classes and each class has index $q^{s}$ with coefficient 1 .

Therefore, for $s \geq 2, \ell \geq 2$ we conclude that:

$$
\begin{aligned}
o\left(q^{s}\right) & =o\left(q^{s-1}\right)+(3.16) \\
& +\sum_{v=1}^{s-\delta-1} \Gamma(s, s-v, v)+\frac{p\left(p^{q^{\delta}}-1\right) q^{s-\delta-1}(q-1)}{p(p-1) q^{\delta}}
\end{aligned}
$$

where $s-\delta-1=\min \{\ell-1, s-1\}$ and $\delta=\max \{0, s-\ell\}$.

If $\ell=1$ then we have:

$$
0\left(q^{s}\right)=0\left(q^{s-1}\right)+(3.16)+\frac{p\left(p^{q-1}-1\right)(q-1)}{p(p-1) q^{s-1}} .
$$

\section{RFFERENCES}

1. LIANG, Joseph J., On the solutions of trinomial equations over a Finite Field. Bull. Calcutta Math. Soc. 70(1978), no. 6, pp. 379-382.

2. LONG, Andrew F., Factorization of irreducible Polynomials over a Finite Field with the substitution X9-X for X. Acta Arith. XXV 1973, pp. 65-80.

3. ALBERT, A.A., Fundamental Concepts of Higher Algebra. Phoenix Science Series, The University of Chicago Press, 1956.

4. ALAHEN, J.D. and KNUTH, Donald E., Tables of Finite Fields. Sankhya, The Indian Journal of Statistics, Series A, Vol. 26, Dec. 1964, Part 4, pp. 305-328. 


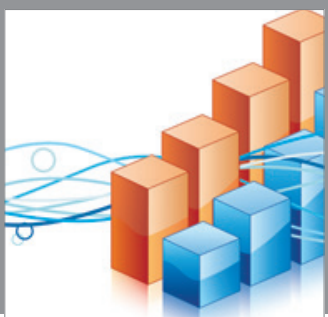

Advances in

Operations Research

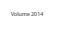

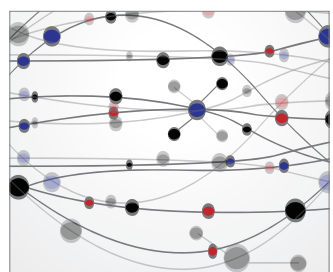

\section{The Scientific} World Journal
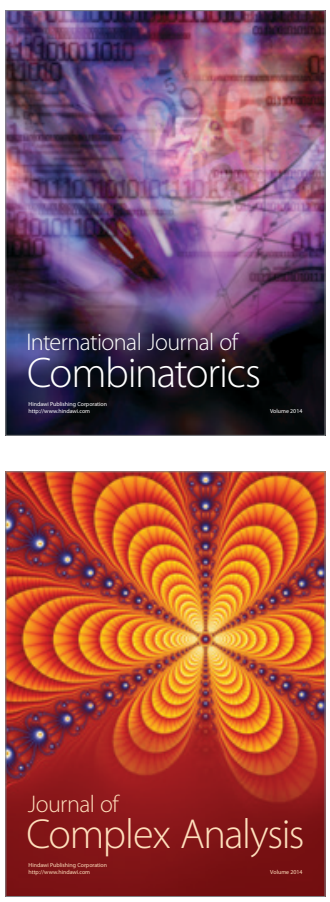

International Journal of

Mathematics and

Mathematical

Sciences
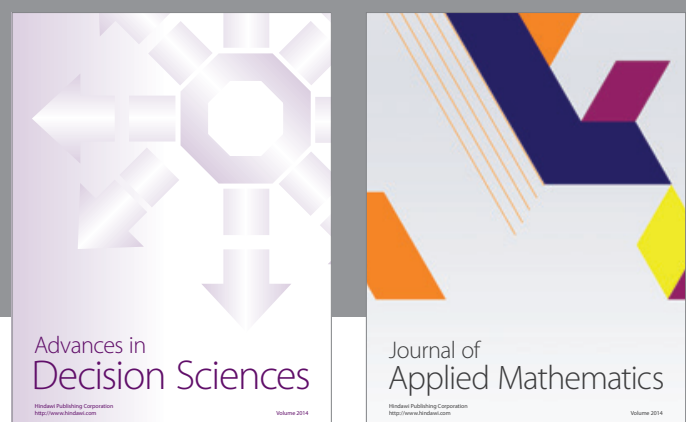

Journal of

Applied Mathematics
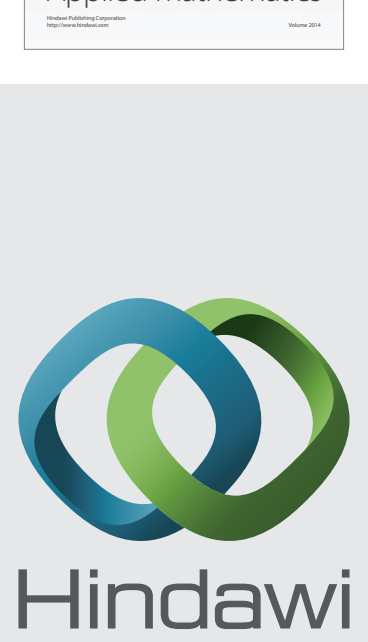

Submit your manuscripts at http://www.hindawi.com
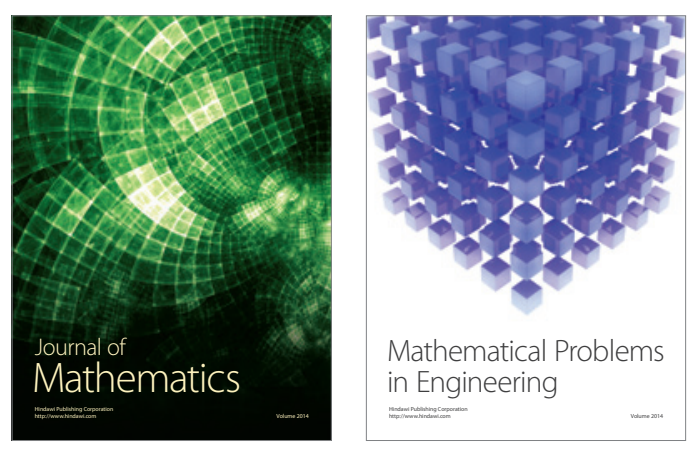

Mathematical Problems in Engineering
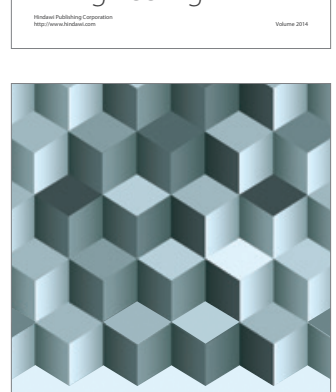

Journal of

Function Spaces
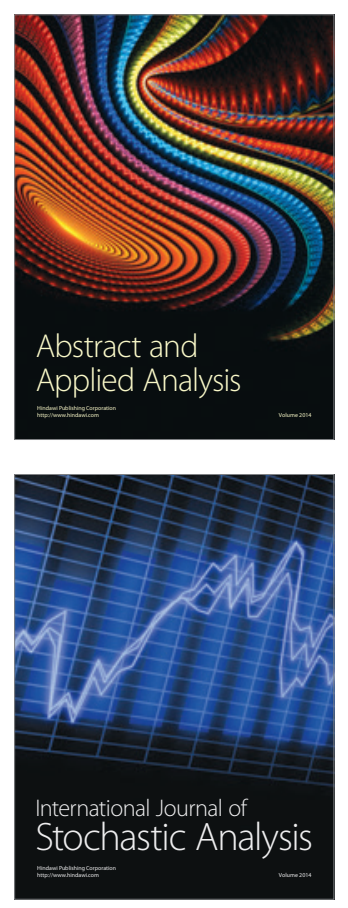

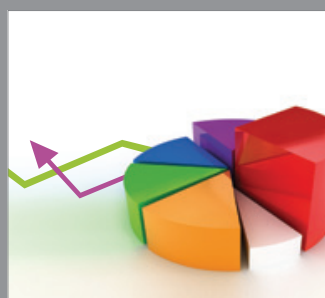

ournal of

Probability and Statistics

Promensencen
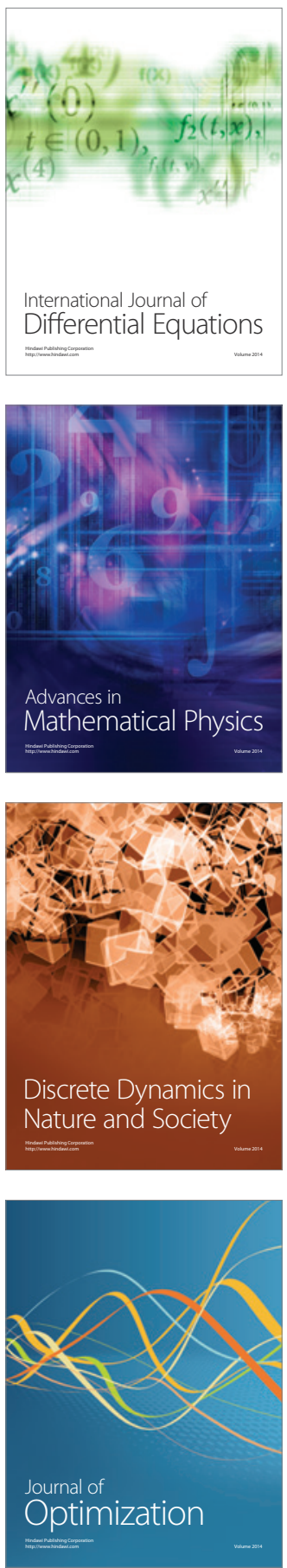